\title{
Über die Verbindungen der Protamine mit anderen EiweiBkörpern.
}

\author{
Von \\ Androw Hunter, Carnegie Research Fellow. \\ (Aus dem physiologischen Institut der Universität Heidelberg.) \\ (Der Redaktion zugegangen am 27. Oktober 1907.)
}

Im Jahre 1894 machte A. Kossel die Beobachtung, 1) daß gewisse Protamine sich in schwach alkalischer Lösung mit anderen Eiweißkörpern unter Bildung eines Niederschlages vereinigen. Diese Reaktion zeigte sich beim Salmin, Clupein, Cyclopterin, Scombrin und Sturin. Sie vollzieht sich beim Zusammentreffen der beiden Eiweißkörper in schwach alkalischer Lösung bei gewöhnlicher Temperatur und führt zur Bildung eines Produktes, welches einem natürlich vorkommenden Eiweißkörper, dem Histon, sehr ähnlich ist. A. Ko ssel äußerte daher sogleich die Vermutung, daß das Histon als eine Vereinigung von Protamin mit einem komplizierteren Eiweißkörper aufzufassen sei. Diese Vermutung wurde um so wahrscheinlicher, als die späteren Untersuchungen von A. Kossel und F. Kutscher ${ }^{2}$ ) das Histon als einen Eiweißkörper definiert hatten, welcher sich durch einen hohen Gehalt an basischen Bausteinen auszeichnet und in dieser Hinsicht eine Mittelstellung zwischen den Protaminen und den höheren Eiweißsubstanzen einnimmt.

Hingegen führten die neueren Untersuchungen über die nächsten Spaltungsprodukte des Histons zu dem Ergebnis, daß dieser Körper keinenfalls mit der loekeren, bei der Vereinigung von Protamin und Eiweiß direkt entstehenden Verbindung identisch sein kann, denn das Histon zerfällt bei der Pepsinverdauung unter Bildung eines charakteristischen Körpers, des "Histopeptons», ${ }^{3}$ ) während die künstlich hergestellte Verbin-

1) Deutsche medizinische Wochenschrift, 1894, Nr. 7.

2) Diese Zeitschrift, Bd. XXXI, S. 188 u. f.

s) Diese Zeitschrift, Bd. XLIX, S. 308. 
dung von Clupein oder Sturin mit Eiweiß - wie mir Herr Professor Kossel nach seinen Beobachtungen mitteilte - bei derselben Einwirkung wieder unter Rückbildung von Protamin zerlegt wird. Wenn also das Histon überhaupt durch die Anlagerung eines protaminartigen Körpers an einen anderen Eiweißstoff entstände, so müßten in dem Vereinigungsprodukt noch Umlagerungen unbekannter Art vor sich gehen, wie dies schon in der ersten Mitteilung von A. Kossel in Erwägung gezogen ist. ${ }^{1}$ )

Die soeben angeführte Beobachtung über die glatte Rückbildung des Protamins aus seiner Eiweißverbindung zeigt nun den Weg zu einer Feststellung der Mengenverhältnisse, in welchen sich Protamin und andere Eiweißkörper vereinigen. Auf Veranlassung des Herrn Professor Kossel habe ich einige Untersuchungen in dieser Richtung angestellt.

Es ist bisher nichts bekannt über den chemischen Mechanismus solcher Vereinigungen verschiedener Eiweißkörper untereinander oder über diejenige Atomgruppierung im Eiweißmolekül, welche diese Verbindungen vermittelt. Nur das eine weiß man: daß es eine bei der Hydrolyse sehr leicht zerstörbare Gruppe sein muß. Denn wenn man das Clupein durch halbstündiges Erwärmen mit 10\% iger Schwefelsäure in Clupeon überführt, so ist die Fähigkeit, mit Eiweiß Niederschläge zu bilden, vernichtet. ${ }^{2}$ ) Zugleich ist auch die bemerkenswerte Wirkung des Protamins auf den Blutdruck verschwunden. ${ }^{3}$ ) Ebenso kann andererseits auch ein komplizierterer Eiweißkörper durch die Hydrolyse leicht so weit verändert werden, daß er nicht mehr mit Protamin reagiert.

Bei folgenden Eiweißsubstanzen konnte ich in schwach ammoniakalischer Lösung eine Fällung mit Clupein erzielen:

krystallisiertes Eieralbumin,

Casein,

Hemielastin,

Leim,

1) Deutsche medizin. Wochenschrift, 1894 (1. c.)

2) A. Kossel, Diese Zeitschrift, Bd. XXV, S. 174; M. Goto, Diese Zeitschrift, Bd. XXXVH, S. 106.

s) W. H. Thompson, Diese Zeitschrift, Bd. XXIX, S. 1. 
Edestin,

Heteroalbumose,

Protalbumose.

Ferner wurde *Alkalialbuminat (aus Ochsenfleisch) in der durch einen geringen Überschuß von Natron bewirkten Lösung, Histonsulfat bei Gegenwart von wenig Natriumcarbonat untersucht. In beiden Fällen trat Niederschlagbildung ein.

Hingegen erzielte ich in schwach ammoniakalischer Lösung keine Fällung mit:

\section{Elastinpepton, Deuteroalbumose, ${ }^{1}$ ) \\ Histopepton.}

In dem gewöhnlich als "Deuteroalbumose» bezeichneten Gemisch scheinen zuweilen auch Stoffe vorzukommen, welche einen im Überschuß des Clupeins und der Albumose sehr leicht löslichen Niederschlag ergeben.

Außerdem untersuchte ich noch einige Polypeptide, welche ich der Freundlichkeit des Herrn Dr. E. Abderhalden verdanke und zwar

Glycylalanin, Leucylglycin,

Phenylalanin-leucin,

Glycyl-l-tyrosin,

Diglycylglycin,

Alanylglycylglycin,

Triglycylglycin.

Keines dieser Präparate gab die charakteristische Reaktion mit Protamin. ${ }^{2}$ )

\section{Methode der Untersuchung.}

Die Methode beruht auf der Eigenschaft der Protamine, durch pikrinsaures Natrium in schwach alkalischer Lösung

1) Aus Fibrin, Syntonin, Casein nach Folin (Diese Zeitschrift, Bd. XXV, S. 152) dargestellt. Niederschlag.

2) Das Clupein gibt - wie ich fand - auch mit Gallensäuren einen 
quantitativ gefällt zu werden, während Albumosen unter den gewählten Bedingungen in Lösung bleiben, und ferner auf der Tatsache, daß die Verbindung «Protamin-Eiweiß» durch Pepsinverdauung in ihre Komponenten gespalten wird.

Demnach wird der zu untersuchende Körper mit einer Pepsin-HCl-Lösung (deren Stickstoffgehalt extra nach Kjeldahl ermittelt wird) acht Tage bei $36^{\circ}$ verdaut. Dadurch wird das Protamin in Freiheit gesetzt und der andere Komponent in Deuteroalbumosen und Peptone verwandelt.

Darauf wird nach eventuellem Filtrieren die Verdauungsflüssigkeit durch $\mathrm{NaOH}$ schwach alkalisch gemacht. Neutralisieren genügt nicht, denn bei streng neutraler Reaktion werden auch manche Deuteroalbumosen teilweise durch Natriumpikrat gefällt; anderseits darf die Alkalescenz nicht zu stark werden, sonst wird das Protamin nicht vollständig ausgefällt. Um genaueste Resultate zu bekommen, muß die Flüssigkeit gegen Lackmus eben schwach alkalisch sein.

Dabei scheidet sich gewöhnlich ein geringer Niederschlag aus, wahrscheinlich eine Fällung kleiner Reste von primären Albumosen durch das Protamin. Sein Stickstoffgehalt ist gewöhnlich nur gering. In den folgenden Versuchen wird er angegeben, aber bei der Berechnung der Resultate nicht in Betracht gezogen, da eine einfache Erwägung ergibt, daß das Resultat durch seine Berücksichtigung nicht wesentlich geändert wird. Die nötigenfalls von dieser Abscheidung abfiltrierte Lösung wird nun auf ein bestimmtes Volumen (z. B. $200 \mathrm{ccm}$ ) gebracht und der Stickstoff in einem genau abgemessenen Teil (z. B. $10 \mathrm{ccm}$ ) zweimal bestimmt. Nach Abzug des in der Pepsinlösung vorhandenen Stickstoffs bekommt man also den ganzen Stickstoff der untersuchten Verbindung.

Dann wird ein anderer aliquoter Teil (z. B. $50 \mathrm{ccm}$ ) sorgfältig mit der nötigen Menge Natriumpikrat gefällt. Ein Überschuß wirkt etwas lösend und muß vermieden werden. Der Niederschlag wird auf einem stickstofffreien Filter gesammelt und mit destilliertem Wasser gut gewaschen. Filter samt Niederschlag werden dann zusammen mit schwefelsäurehaltigem Wasser in den Ätherextraktionsapparat gebracht und von Pikrinsäure 
befreit. Die so erhaltene Lösung von schwefelsaurem Protamin wird auf ein bestimmtes Volumen gebracht und in einem aliquoten Teil der Stickstoff bestimmt; oder es wird, wenn nur wenig Protamin vorhanden ist, das Ganze auf einmal nach Kjeldahl verarbeitet. Man erhält so die Menge des durch Natriumpikrat fällbaren Stickstoffs, der als Protaminstickstoff berechnet wird.

Um schließlich die Reinheit des so gewonnenen Protamins $\mathrm{zu}$ kontrollieren, wird der Rest der Verdauungsflüssigkeit mit pikrinsaurem Natrium gefällt, der Niederschlag gesammelt, sorgfältig gewaschen, mit $\mathrm{H}_{2} \mathrm{SO}_{4}$ und Äther von Pikrinsäure befreit und mit Alkohol gefällt. Der mit Alkohol und Äther gewaschene und getrocknete Niederschlag wird dann auf die Reaktionen des Clupeins geprüft.

Ich versuchte auch die Menge des Protamins durch Wägung des Pikrats zu ermitteln. Dieses Verfahren führte jedoch nicht zu zuverlässigen Ergebnissen.

\section{Prüfung der Methode.}

Die Zuverlässigkeit der Methode wurde folgendermaßen geprüft.

Vorversuch I: a) Ungefähr $1 \mathrm{~g}$ Clupeinsulfat wurde in Wasser gelöst und die Lösung auf genau $100 \mathrm{ccm}$ gebracht. In je $10 \mathrm{ccm}$ wurden dann zwei Kjeldahlbestimmungen ausgeführt

Verbrauchte 1/10-Normallösung 13,6 ccm.

b) $20 \mathrm{ccm}$ derselben Lösung wurden neutralisiert und mit Natriumpikrat gefällt. Der Niederschlag im Ätherextraktionsapparat mit $\mathrm{H}_{2} \mathrm{SO}_{4}$ zerlegt und von Pikrinsäure befreit. Die Protaminlösung sodann auf $100 \mathrm{ccm}$ gebracht und in zwei Portionen von je $50 \mathrm{ccm}$ der Stickstoff bestimmt.

\section{Verbrauchte n/10-Lösung 13,65}

Nach Versuch a) berechnet 13,6.

c) $40 \mathrm{ccm}$ der Lösung mit $1 \mathrm{~g}$ Wittepepton und $0,1 \mathrm{~g}$ Pepsin $+4 \mathrm{ccm}$ starker Salzsäure versetzt und bis auf $50 \mathrm{ccm}$ gebracht.

Die Mischung wurde 8 Tage verdaut. Darauf $20 \mathrm{ccm}$ neutralisiert und mit Natriumpikrat gefällt. Der Niederschlag 
wie vorher behandelt und der Stickstoff in je $10 \mathrm{ccm}$ bestimmt.

Verbrauchte n/10-Lösung (Mittel) 11,55

Berechnet nach Versuch a) 10,88

Somit Fehler

$+5,7 \%$.

Vorversuch II. a) Ungefähr 1 g Clupeinsulfat in Wasser gelöst und auf $100 \mathrm{ccm}$ gebracht.

n/10-Lösung für je $10 \mathrm{ccm}$ verbraucht: 15,95 .

b) $20 \mathrm{ccm}$ derselben Lösung schwach alkalisch gemacht und mit Natriumpikrat gefällt.

Aus dem Niederschlag wurde wie beim ersten Versuch der Stickstoff wieder gewonnen und zwar:

Verbrauchte n/10-Lösung $\quad 15,45 \mathrm{ccm}$

Nach Versuch a) berechnet 15,95 》

c) $40 \mathrm{ccm}$ der Lösung mit $1 \mathrm{~g}$ Wittepepton und $0,1 \mathrm{~g}$ Pepsin $+0,4 \mathrm{ccm}$ starker $\mathrm{HCl}$ versetzt und 4 Tage verdaut. Dann auf $50 \mathrm{ccm}$ gebracht.

Davon $20 \mathrm{ccm}$ schwach, aber deutlich alkalisch gemacht und mit Natriumpikrat gefällt. Gefällter Stickstoff wie früher bestimmt.

Verbrauchte Kubikzentimeter n/10-Lösung 13,3

Nach Versuch a) berechnet

Somit Fehler

$$
=+4,2 \% \text {. }
$$

Aus diesen Versuchen geht zugleich hervor, daß das Protamin mit Deuteroalbumosen resp. Peptonen keine Verbindung gibt. Es wäre nämlich denkbar, daß Protamin, obgleich es keine Fällung mit Deuteroalbumosen gibt, doch mit denselben in lösliche Verbindungen eintritt. Wenn das aber der Fall wäre, müßte diese Verbindung mit dem Protamin gefällt werden und den Protaminstickstoff beträchtlich erhöhen. Eine Übereinstimmung innerhalb der von mir gefundenen Grenze wăre dann nicht möglich.

Untersuchung der Protamin-Eiweißverbindungen.

Untersucht wurden die Verbindungen von Clupein mit Syntonin, Eieralbumin, Leim, Hemielastin, Casein und Edestin.

\section{Clupein-Syntonin.}

Aus Ochsenfleisch wurde eine klare salzsaure Lösung von «Syntonin» dargestellt. Dazu wurde soviel Natronlauge hinzugefügt, daß die voluminöse Fällung wieder in Lösung ging. Die 
Alkalescenz der Lösung war nün eine derartige, daß von $10 \mathrm{ccm}$ $1,25 \mathrm{ccm}$ n/10-Oxalsäure neutralisiert wurden. Die Lösung enthielt ca. 1,5\% Eiweiß.

$1 \mathrm{~g}$ Clupeinsulfat wurde in Wasser gelöst, und die alkalische Syntoninlösung hinzugefügt, so lange noch ein Niederschlag entstand.

Der flockige Niederschlag ist in Überschuß von Clupein leicht löslich, in Überschuß von Syntonin nicht, oder wenig löslich. Nach der Fällung war die Alkalescenz geringer; die $10 \mathrm{ccm}$ entsprechende Flüssigkeitsmenge sättigte jetzt nur $0,55 \mathrm{ccm} \mathrm{n} / 10-0 x a l s a ̈ u r e$.

Der Niederschlag wurde abfiltriert, mit Wasser, Alkohol und Äther gewaschen und getrocknet. Ausbeute $3,1 \mathrm{~g}$.

$2,8 \mathrm{~g}$ wurden mit $100 \mathrm{ccm}$ Pepsinsalzsäure $(0,1 \mathrm{~g}$ Pepsin auf $150 \mathrm{ccm} 0,8 \% \mathrm{HCl}) 6$ Tage verdaut. Darauf wurde die klare Lösung mit Natronlauge schwach alkalisch gemacht. Dabei entstand ein Niederschlag, der abfiltriert wurde; er enthielt soviel Stickstoff, wie $13,95 \mathrm{ccm} \mathrm{n} / 10-\mathrm{NH}_{3}$ entspricht.

Das klare Filtrat wurde auf $200 \mathrm{ccm}$ gebracht, welche nach dem oben angegebenen Verfahren untersucht wurden. Die gefundenen Stickstoffmengen - in Kubikzentimetern ZehntelNormallösung ausgedrückt - sind folgende.

Stickstoff in $200 \mathrm{ccm}$ der Lösung 351

Davon entfallen auf die angewandte Pepsinlösung 10

Also im Protamineiweiß 341

Durch Pikrat fällbare Substanz 117,2

Das Verhältnis des Gesamtstickstoffs zum Protaminstickstoff beträgt daher $100: \mathbf{3 4 , 3 6}$.

Aus dem Rest des Filtrats wurde, wie früher beschrieben, das Clupein wiedergewonnen. Es erwies sich als fast vollständig rein, wie aus den folgenden Reaktionen sichtbar ist.

\begin{tabular}{|c|c|}
\hline Ammoniak & Trübung \\
\hline Wittepepton & Niederschlag \\
\hline Biuretreaktion & vorhanden \\
\hline Ferrocyankalium in neutraler Lösung & Niederschlag \\
\hline Millonsche Reaktion & fehlt vollständig \\
\hline $\left.\begin{array}{l}\text { Schwefelbleireaktion } \\
\text { Glyoxylsäurereaktion }\end{array}\right\}$ & minimale Spur. \\
\hline
\end{tabular}




\section{Clupeineieralbumin.}

Eine ammoniakhaltige Lösung des krystallisierten Eieralbumins wurde mit $1,5 \mathrm{~g}$ Clupeinsulfat in stark ammoniakalischer Lösung gefällt.

Die Verbindung fällt in Form kleiner flüssiger Tropfen aus, die nach eintägigem Stehen ein klares, fast farbloses Öl am Boden des Gefäßes bilden. Das Öl ist in Überschuß von. Albumin löslich. Es kann auch in verdünnter HCl gelöst werden, und wird durch Ammoniak wieder ausgefällt. Diese Eigenschaft wurde zur Reinigung des Produktes benutzt. Dann wurde es unter Alkohol und Äther erhärtet und gepulvert. Es stellte so ein ganz weißes Pulvèr dar.

Ausbeute 6,1 g.

$6 \mathrm{~g}$ wurden mit $150 \mathrm{ccm}$ Pepsin-HCl 9 Tage verdaut und dann schwach alkalisch gemacht. Der entstehende flockige Niederschlag, welcher sehr gering war, enthielt eine $8,7 \mathrm{ccm}$ n/10-Lösung entsprechende Stickstoffmenge. Das Filtrat wurde auf $200 \mathrm{ccm}$ gebracht und genau wie früher beschrieben behandelt.

Gesamtstickstoff des Filtrats (minus Pepsinstickstoff) = 621 ccm n/10-Lösung.

Durch Natriumpikrat fällbarer Stickstoff $182 \mathrm{ccm}=$ $\mathbf{2 9 , 3 1} \%$.

Reaktionen des wiedergewonnenen Clupeins:

$\left.\begin{array}{l}\text { Millonsche Reaktion } \\ \text { Hopkins } \\ \begin{array}{l}\text { Schwefel- } \\ \text { Wittepepton }\end{array}\end{array}\right\} \begin{aligned} & \text { vollständig negativ. } \\ & \text { deutlicher Niederschlag. }\end{aligned}$

\section{Clupeinleim.}

Leimlösung: $5 \mathrm{~g}$ Leim, $210 \mathrm{ccm}$ ammonhaltiges Wasser.

$110 \mathrm{ccm}$ dieser Lösung durch $0,5 \mathrm{~g}$ Clupeinsulfat gefällt. Dann mit Wasser verdünnt (wobei der Niederschlag sich etwas vermehrt) und mit Äther zusammengeschüttelt. Der Niederschlag. ballt sich jetzt zusammen, er kann leicht abfiltriert und mit 1\% wässeriger Ammoniaklösung gewaschen werden.

Der Körper hat eine gallertartige Beschaffenheit. Seine Eigenschaften weichen erheblich von denen der andern beschrie- 
benen Körper ab. Er ist in heißem Wasser leicht, in warmer, konzentrierter Kochsalzlösung äußerst leicht löslich. Wenn die Lösung nicht zu salzarm und nicht zu salzreich, nicht zu konzentriert und nicht $\mathrm{zu}$ verdünnt ist, fällt er beim Erkalten wieder flockig aus. Wenn aber keine Salze oder zuviel Salze vorhanden sind, besonders wenn die Lösung sehr verdünnt ist, kann er sehr langsam oder garnicht ausfallen; in diesem Fall kann man gewöhnlich eine Abscheidung erzielen, wenn man eine Spur NaCl (2 Tropfen einer gesättigten Lösung auf $100 \mathrm{ccm}$ ) zugibt, und diese Abscheidung wird dann durch $\mathrm{Zu}-$ satz von $\mathrm{NH}_{3}$ noch bedeutend vermehrt.

Wenn der Körper dagegen in zu konzentrierter Lösung ist, fällt er zwar zum Teil flockig aus, zum Teil aber auch als Öl; das Öl sinkt dann zu Boden und erstarrt wie Gelatine. Eine sehr konzentrierte Lösung kann sogar beim Abkühlen vollständig leimartig erstarren.

Zur Reinigung des Körpers verfährt man demnach am besten in der Weise, daß man ihn in einer großen Menge warmen, destillierten Wassers löst, eine Spur $\mathrm{NaCl}$ und ein paar Tropfen $\mathrm{NH}_{3}$ hinzufügt und abkühlen läßt. Der größte Teil fällt dann flockig aus. Wenn jedoch ein Teil gelatiniert, muß das Ganze wieder in mehr Wasser aufgelöst und in derselben Weise gefällt werden.

Ferner ist der Körper in kalten verdünnten Säuren löslich, obgleich schwer, und nur in Überschuß vollständig. Auf Zugabe von $\mathrm{NH}_{3}$ wird er nicht immer wieder ausgefällt, vielleicht infolge der Salzbildung.

In warmen verdünnten Säuren ist er leicht löslich; die Lösung erstarrt beim Abkühlen wie Leim, wahrscheinlich führt jedoch diese Behandlung bereits eine Zersetzung der Verbindung herbei. Die Ausbeute an gereinigtem Produkt betrug nach dem Trocknen mit Alkohol und Äther 1,6 g. Die Substanz stellte ein weißes Pulver dar.

1,55 g wurden mit $50 \mathrm{ccm}$ Pepsin-HCl 10 Tage verdaut und dann schwach alkalisch gemacht. Dabei entstand kein Niederschlag.

Gesamtstickstoff (minus Pepsin) $=194 \mathrm{ccm}$. 
Durch Pikrat fällbar $44,2 \mathrm{ccm}$.

Das Verhältnis des Protaminstickstoffs zum Stickstoff der ganzen Verbindung betrug also $=\mathbf{2 2 , 7 9} \%$.

Das weiter gewonnene Clupein zeigte die Reaktionen des Clupeins, keine Millonsche Reaktion.

\section{Clupein-Hemielastin.}

Unter Zusatz von $\mathrm{NH}_{3}$ wurde eine Hemielastinlösung durch 0,8 g Clupeinsulfat gefällt. Die Fällung war zuerst ölig und wandelte sich beim Stehen in eine zähe feste Masse um.

Dieselbe ist in kaltem und heißem Wasser, kalten Säuren und Salzlösungen unlöslich. Durch siedende Säuren wird sie langsam angegriffen und dann nur unvollständig wieder von $\mathrm{NH}_{3}$ gefällt. Durch heiße Natronlauge wird sie nur sehr schwer und langsam aufgelöst. Nachdem diese Masse so gut wie möglich durch Anreiben mit Wasser gereinigt war, wurde sie mit Alkohol und Äther getrocknet und gepulvert: Ausbeute 2,2 g.

$2,1 \mathrm{~g}$ wurden mit $75 \mathrm{ccm}$ Pepsinsalzsäure 9 Tage verdaut, wobei die Substanz bis auf ganz kleine Reste aufgelöst wurde. Die Flüssigkeit wurde dann schwach alkalisch gemacht, wobei ein sehr geringer Niederschlag ausfiel, dessen Stickstoffgehalt $=2,15 \mathrm{~cm} \mathrm{n} / 10$-Lösung entsprach. Die filtrierte Flüssigkeit gab nun beim Kochen nur noch eine kleine Trübung (unverändertes Hemielastin). Sie wurde auf $200 \mathrm{ccm}$ gebracht, und nach der beschriebenen Methode analysiert.

Gesamtstickstoff (minus Pepsin) 235,1 ccm, wovon durch Pikrat fällbar $53,4 \mathrm{ccm}$.

Es war hiernach 22,71\% des Gesamtstickstoffs der Verbindung in Form von Protaminstickstoff enthalten.

Das wiedergewonnene Protamin verhielt sich wie beim vorigen Versuch.

\section{Clupein-Casein.}

Eine ammoniakalische Lösung von Casein wurde mit $1 \mathrm{~g}$ Clupeinsulfat (auch in ammoniakalischer Lösung) gefällt. Die flockige Ausfällung ballt sich zu einer zähen Masse zusammen. 
Die Verbindung ist in kaltem Wasser unlöslich, in heißem sehr schwer löslich; in verdünnten Säuren etwas löslich, besonders beim Erhitzen, und fällt dann (wenn nicht zu viel Salz gebildet ist) auf Zusatz von $\mathrm{NH}_{3}$ wieder aus. Sie ist in Kochsalzlösung, besonders beim Erhitzen, löslich.

Sie wurde durch Anreiben mit Wassèr gereinigt, getrocknet und gepulvert. Ausbeute $2,8 \mathrm{~g}$.

2,6 $\mathrm{g}$ wurden mit $125 \mathrm{ccm}$ Pepsin-HCl 8 Tage verdaut. Die Substanz ging nur langsam in Lösung und ein Teil blieb zum Schluß ganz ungelöst. Dieser Rückstand (Paranuclein?) wurde abfiltriert; es enthielt so viel Stickstoff, wie $12,75 \mathrm{ccm}$ n/10-Lösung entsprach.

Das Filtrat wurde schwach alkalisch gemacht und auf $200 \mathrm{ccm}$ gebracht. Dabei wurde es opalisierend und nach einem Tage fiel eine kleine Menge eines klaren Öls aus, welches wohl als eine Verbindung von Protamin und Protocaseose aufzufassen ist. Es enthielt soviel Stickstoff, wie $22,7 \mathrm{ccm} \mathrm{n} / 10-$ Lösung entspricht. Die vom Niederschlag befreite Flüssigkeit wurde wie früher behandelt.

Gesamtstickstoff (minus Pepsin) entspr. $291 \mathrm{ccm} \mathrm{n/10-}$ Lösung, wovon durch Pikrat fällbar entspr. $119 \mathrm{ccm}$.

Der Protaminstickstoff verhält sich zum Gesamtstickstoff hiernach wie 40,88:100.

Wenn man das Paranuclein, das wohl kein Protamin in Verbindung hält, mit hinein zum Gesamtstickstoff rechnet, dann berechnet sich das Verhältnis auf 39,17: 100.

Das wiedergewonnene Clupein gab die typischen Protaminreaktionen, keine Millonsche, keine Schwefelbleireaktion, spurenweise Glyoxylreaktion.

\section{Clupein-Edestin.}

Das Edestin wurde durch $\mathrm{NaCl}$ und viel $\mathrm{NH}_{3}$ in Lösung gehalten und mit $0,5 \mathrm{~g}$ Clupeinsulfat gefällt. Der Niederschlag ist im Überschuß weder von Clupein noch von Edestin erheblich löslich, er ist auch unlöslich im Überschuß von $\mathrm{NH}_{3}$, in heißem Wasser, in Säuren und in Kochsalzlösung. In $\mathrm{NaOH}$ ist er löslich, von $\mathrm{NH}_{4} \mathrm{Cl}$ wieder ausgefällt. 
Das zuerst Ausfallende war gelblich und zäh; der Rest (der größte Teil) flockig und weiß. Nur das Flockige wurde bearbeitet. Es ließ sich leicht abfiltrieren und wurde wiederholt mit ammoniakhaltigem Wasser angerieben und zentrifugiert, um es vom Edestin zu reinigen. Dann wurde es mit. Wasser gewaschen, mit Alkohol und Äther getrocknet. Die Ausbeute betrug $3,6 \mathrm{~g}$ bis zur völligen Entfernung des Ammons.

$3,5 \mathrm{~g}$ wurden 8 Tage mit $100 \mathrm{ccm}$ Pepsinsalzsäure verdaut und ging hierbei bis auf minimale Reste in Lösung. Darauf wurde die Lösung schwach alkalisch gemacht, wobei ein geringer flockiger Niederschlag ausfiel.

Der Stickstoffgehalt des Niederschlages entsprach 17,55 ccm n/10-Lösung.

Der Gesamtstickstoff des Filtrats entsprach $446 \mathrm{ccm}$ n/10Lösung, wovon durch Pikrinsäure fällbar 62,2 ccm.

Somit ist 13,95\% des gesamten Stickstoffs in Form von Protamin enthalten.

Das zurückgewonnene Clupein gab keine Millonsche Reaktion, minimale Spuren von Schwefelbleireaktion und Glyoxylsäurereaktion.

\section{Schlußbemerkungen.}

Wir sehen aus diesen Untersuchungen, daß Eiweißkörper durchaus verschiedener Zusammensetzung mit Protamin reagieren; aber die entstandenen Verbindungen zeigen je nach der Natur des mit Clupein verbundenen Eiweißstoffs verschiedene Eigenschaften. Besonders weicht das Leimprotamin in seinen Löslichkeitsverhältnissen von den übrigen Produkten ab. Auch die Mengenverhältnisse, in welchen sich die beiden Komponenten verbinden, sind durchaus verschiedene. Es ergibt sich dies wohl am besten aus dem Stickstoffanteil, welcher auf jeden der Komponenten entfällt; doch ist es nicht ohne Interesse, auch die Gewichtsmengen in Betracht zu ziehen, die sich bei den meisten Eiweißkörpern leicht aus den Stickstoffmengen berechnen lassen. 
Andrew Hunter, Über die Verbindungen der Protamine.

\begin{tabular}{c|c|c}
\hline $\begin{array}{c}\text { Mit Clupein } \\
\text { verbundener } \\
\text { Eiweißkörper }\end{array}$ & $\begin{array}{c}\text { Auf 100 Teile Stickstoff } \\
\text { der Protamin-Eiweiß-Ver- } \\
\text { bindung sind im Protamin } \\
\text { enthalten }\end{array}$ & $\begin{array}{c}\text { Gewichtsmengen der } \\
\text { Eiweißkörper, welche mit } \\
1 \text { Gewichtsteil Clupein in } \\
\text { Verbindung getreten sind }\end{array}$ \\
\hline Alkalialbuminat . & 34,36 & - \\
Ovalbumin . . . & 29,31 & 4,1 \\
Leim . . . . . & 22,79 & 4,8 \\
Hemielastin . . . & 22,71 & 5,2 \\
Casein . . . . & 39,17 & 2,5 \\
Edestin . . . . & 13,95 & 8,5 \\
\end{tabular}

Die Kosten dieser Untersuchung wurden durch eine Bewilligung vonseiten des Carnegie-Trusts bestritten.

Zum Schluß sage ich Herrn Professor Kossel meinen besten Dank, sowohl für die freundliche Überlassung des Themas, wie auch für den Rat, mit dem er mich bei der Ausführung der Arbeit dauernd unterstützte. 